\title{
The Problem of Identity and a Justification for Non-Reflexive Quantum Mechanics
}

\author{
Décio Krause*
}

Research Group in Logic and Foundations

Department of Philosophy

Federal University of Santa Catarina

www. cfh.ufsc. br/ dkrause

(January 2011)

\begin{abstract}
In this paper we try to justify our way of looking for an alternative approach to quantum mechanics, which is based on a non-classical logic. We consider two specific questions related to quantum theory, namely, entanglement and the indiscernibility of quanta. We characterize individuals, and then explain in what sense entanglement is a concept which can be applied to individuals in a restricted sense only. Then, we turn to indiscernibility and, after realizing that this concept is of a fundamental importance, we mention the 'traditional' theory of identity (TTI) of standard logic and mathematics, which underly the basic formalism of quantum theory. Then we propose to call the Problem of Identity the question whether identity of objects can be justified, and under what conditions. As in the Hume's celebrated Problem of Induction, we conclude that the attribution of transtemporal identity to an object (either a macroscopic or a microscopic one) has no logic justification, and must be considered as a metaphysical hypothesis. Numerical identity is also put aside for similar reasons. Then we guess that identity is just an useful concept, but which in certain fields, mainly in the quantum realm, could be substituted by a weaker concept of indiscernibility. This assumption motivates us to look for an interpretation of quantum mechanics based on a non-classical logic, termed non-reflexive, and the corresponding mechanics is called non-reflexive quantum mechanics.
\end{abstract}

Keywords: non-reflexive logic, foundations of quantum mechanics, entanglement, non-individuality, quasi-sets, identity of objects, indiscernibility.

Introduction Many time ago, Protagoras guessed that "Man is the measure of all things". As usually read, this dictum (apparently) says that things are just invention of men and, in some sense, there is no reality out of phenomena. But there might be a second reading of his claim; things and their 'measures' are just schemes we can form of an independent reality, whose existence we don't need to put aside but whose 'real nature' remains veiled to us (cf. [d'Es.83, p.19]). The most successful 'schemes' are of course those advanced by the relevant physical theories, and if we embark in a 'naturalized' metaphysics, ${ }^{1}$ agreeing that it would be suspect to read our ontology off our the best theories, we should acknowledge

\footnotetext{
* Partially supported by CNPq (300122/2009-8).

1. We may take this notion in the following sense: "[b]y this [naturalized metaphysics] we mean a metaphysics that is motivated exclusively by attempts to unify hypothesis and theories that are taken seriously by contemporary science" [Lad.07, p.1].
} 
that "whoever tries to form and idea of the world (...) has to take the findings of quantum physics most seriously into account." [d'Es.03, p.xvi].

Of course we could just dismiss the search for an understanding of the supposed underlying reality as a second order question, as some physicists do; the Nobel Prize winner Stephen Weinberg, for instance, said that "[o]nce again I repeat: the aim of physics at its most fundamental level is not just to describe the world but to explain why it is the way it is." [Wei.93, p.175]. That is, questions of ontology would not concern physics itself. Similar things could be said of the Copenhagen interpretation, mainly in what regard Bohr's and Pauli's stances, to whom the very notion of science does not necessitate of an underlying reality. In fact, as said d'Espagnat, for Bohr science "seems to be a syntesis of that part of human experience that is communicable to any human being. In his view the concept of reality is secondary to such an objective" [d'Es.83, pp.17]. But we as philosophers can of course do otherwise and be interested in looking to the forms of the world these theories face us.

In special, we may turn to some specific questions, and here I underly two of the most distinctive traits of quantum theory, namely, the notion of entanglement, and the indiscernibility of quanta. Firstly we need to explain in what sense entanglement is a concept which can be applied to individuals only in a restricted sense. To do this we need to characterize individuals and circumvent some interpretations of quantum mechanics, such as Bohm's theory (which involves entanglement in a sense and is a theory of individuals). Then, we turn to indiscernibility; after realizing that this concept is of a fundamental importance, we briefly discuss the notion of identity as ascribed by standard logic and mathematics, which underly the basic formalism of quantum theory. Then we propose to call the Problem of Identity the question whether identity of objects (either macroscopic or microscopic) can be logically justified; this is to be taken similarly as Hume's Principle of Induction, namely, the question whether inductive inferences are justified, and under what conditions [Pop.02, p.4]. As in the case of induction, we conclude that the attribution of a permanent identity to an object has no logical justification, and must be considered as a metaphysical hypothesis. This motivates us to look for an interpretation of quantum mechanics based on a nonclassical logic, termed non-reflexive, in which the traditional theory of identity (TT) does not hold in full. The corresponding mechanics is called non-reflexive quantum mechanics.

Entanglement Roughly speaking, entanglement is a property of the quantum state of a system containing at least two objects, which are linked in such a way that we cannot adequately describe the state of any member of the system without mentioning the states of the other members as well, and this is so even if the objects are far away separated. As Schrödinger said when introduced this concept,

"When two systems, of which we know the states by their respective representation, enter into a temporary physical interaction due to known forces between them and when after a time of mutual influence the systems separate again, then they can no longer be described as before, viz., by endowing each of them with a representative of its own. I would not call that one but rather the characteristic trait of quantum mechanics." (apud [Acz.01, p.70])

Hence, once entangled, even if they become apart again, two systems cannot any more be identified by a which is which criterion. The differences to 'classical' objects can be put as follows. Suppose you have two or more white cricket balls which look completely alike. Even you put them together in a container, a trained eye may distinguish one selected ball, and even if you divert your gaze for a moment, in principle you can continue to follow your 
ball, for they are distinct individuals. Contrariwise, if the balls were quantum objects, when merged (technically, when superposed or entangled), you don't have $n$ balls anymore, but a white glob in which there is no more individual elements. Let us explore a little this idea. Firstly, we should realize that quantum mechanics is not just formalism. It encompasses an interpretation. To contrast, we shall consider two of them, namely, the Copenhagen interpretation -the 'standard' interpretation- and Bohm's interpretation. In short, according to the Copenhagen interpretation (there are variants of this view we shall not consider here) quantum mechanics has the following characteristics: (1) the physical state of the considered system is completely described by the wavefunction $|\psi\rangle$; (2) a system possess a certain property only if $|\psi\rangle$ is an eigenvector of the corresponding Hermitian operator representing the property; (3) the wavefunction evolves deterministically according to the Schrödinger equation, but sometimes (4) the wavefunction $|\psi\rangle$ collapses in a way we generally don't now how, and (5) the dynamics is so that if two quantum systems begin in identical states, they may end up in completely different states; we say that the dynamics is stochastic rather than deterministic. In Bohm's theory, the $|\psi\rangle$ function does not provide a complete description of the state, but the system may have other properties, such as position. These additional parameters are called hidden variables; the system has definite properties even if $|\psi\rangle$ is not an eigenvector of the corresponding operator, and the dynamics evolves deterministically, including the hidden variables. Finally, there is no collapse due to previous condition, and systems with the same wavefunction may differ by hidden parameters. The formalisms of the standard view and Bohm's differ a little, but they agree in the relevant parts that interests us here, so that in speaking in the formalism, we can treat them jointly.

According to the formalism, if $\left|\psi_{1}\right\rangle$ describes the state of the first system and $\left|\psi_{2}\right\rangle$ describes the state of the second (note that these wavefunctions label the objects), then the joint system is given by $\left|\psi_{12}\right\rangle=\frac{1}{\sqrt{2}}\left(\left|\psi_{1}\right\rangle\left|\psi_{2}\right\rangle \pm\left|\psi_{2}\right\rangle\left|\psi_{1}\right\rangle\right)$ in the Dirac notation, where, as it is well known, the plus sign holds for bosons while the minus sign holds for fermions. This wavefunction is either symmetric (plus sign) or anti-symmetric (minu sign) under permutation of the two quanta, but its square, namely, $\left|\psi_{12}\right|^{2}$, which gives the relevant probabilities, remains the same, so, the wavefunction $\left|\psi_{12}\right\rangle$ 'confounds' the labels and the quanta are taken as indiscernible, and while this wavefunction continues to express the state of the joint system, no identificacion of the quanta is possible; they got entangled. For instance, the two quantum systems would be the two electrons (which are fermions) of an Helium atom in the fundamental state. There, we know that one of then has spin UP and the another one has spin DOWN in a given direction, but it is impossible (according to standard quantum mechanics) to tell which is which. Furthermore, according to most interpretations of the theory, we cannot even say that there is some hidden parameter which, once known, could provide the distinction. Both in the standard interpretations (Copenhagen view) and in Bohmian mechanics, respectively either there are no such hidden parameters or they remain hidden, and cannot be known. In both cases the impossibility of knowing which is which remains. Let us call an individual whatever object having at least the following characteristics: (i) it has its own peculiar characteristics (properties an relations with other objects), and at least one of them is not partaken by any other individual; (ii) it is a continuant, that is, even if some of its properties and relations change with time, we can still regard them as being the same object as before; (iii) if an individual enter in a crowd with other individuals, even if we cannot even more identify it (such as an ant we were pursuing and which entered the anthill), even so it retains its individuality, being (in principle) distinct from any other individual. We clearly see in these claims the link with the notion of identity.

The ontology of Bohm's theory admits particles on a pair with their classical twins (described by classical physics). These particles have always well defined positions and tra- 
jectories (although hidden), so the theory is compatible with an ontology of individuals, and so Bohmian mechanics is compatible with the idea that the individuality of the underlying entities (pace, Weinberg) exist, yet veiled. The same happens sometimes in standard mathematics, when we know that certain entities are distinct but cannot express the difference by a formula (the case of two least elements of two disjointed sets of real numbers according to some well ordering is a paradigmatic example). ${ }^{2}$ So, we really can keep the standard logic (and mathematics) intact in treating these questions, but at the expenses of introducing hidden parameters. No problem if we adopt such a view. Bohmian mechanics is a fantastic subject, and it is considered as experimentally equivalent with the standard view. But let us turn to our second concept, namely, identity.

Identity and Individuality In quantum physics, the indiscernibility of quantum objects (whatever they are, particles or fields) is a fundamental concept; without assuming it, we cannot obtain the results of quantum mechanics (we cannot revise the reasons here - see [Sch.09] for instance, and below - but just recall the importance of the concept for getting the periodic table of the elements for instance). What is indiscernibility? Well, we may say that two objects $a$ and $b$ are indiscernible relatively to a certain property $P$ if both $a$ and $b$ share $P$, that is, if and only if $P(a) \leftrightarrow P(b)$. This of course can be generalized both to more properties and to $n$-ary relations. We say that $a$ and $b$ are absolutely indiscernible if and only if they share all their properties and relations. Quantum mechanics considers two quantum objects (particles, say) as identical in the first sense, being the selected properties called intrinsic (independent of state), such as electric charge, momentum, and spin. Mathematics treats identity in the second sense; this definition of identity is called Leibniz Law. The two concepts do not exclude one another; once we regard intrinsic properties as some of the properties an object may have, there is always space for other (perhaps 'hidden') properties and relations not considered in the discussion. This is precisely what happens when we treat indiscernibility within a 'classical' framework, such as the Zermelo-Fraenkel set theory (ZF), which encompasses classical logic. ${ }^{3}$

Thus, we can mimic indiscernibility in ZF for instance by means of an equivalence relation and consider the elements of a same equivalence class as indiscernible. But this is just a trick, similar to the assumption of hidden parameters. Really, within ZF, once classical logic holds, any two objects are either identical (in the Leibnizian sense mentioned above) or distinct, although in this last case they may partake some common characteristics (thus being just relatively indiscernible). Technically, we may say that we can consider certain mathematical structures (built in ZF) which are non-rigid so that, once we work within those structures, we can regard some objects as indiscernible relative to all the predicates and relations defined in the structure. This is essentially Hermann Weyl's approach (see [FrKr.06]) and it is consonant with the standard approach to quantum theory either. In fact, once the underlying logic of quantum mechanics is classical logic (basically, ZF), all

2. Since we shall make reference to this example later, let me explain it a little bit more. In ZFC, the ZermeloFraenkel set theory with the Axiom of Choice, we can prove that any set admits a well ordering (a total order so that every non-empty subset has a least element -an element less than any other of the subset); by the way, this proposition is equivalent to the axiom of choice. So, the set $\mathbb{R}$ of real numbers admits a well ordering (really, infinitely many of them), let us call it $R$. Now, let $(0,1)$ and $(2,3)$, given in the usual order, be two disjoined subsets of $\mathbb{R}$. Thus, they have least elements, and of course they are distinct. The problem is that we cannot define the ordering $R$ by a formula of $\mathrm{ZFC}$, so we cannot name these least elements either. Anyway, the standard theory of identity continues to hold, so, we can assure that they are distinct, yet we cannot point the distinction.

3. We shall not discuss here the different possible formulations of ZF; Leibniz Law is a second order expression, and usually $\mathrm{ZF}$ is based on a first-order language. These details were considered elsewhere and do not interfere in the argumentation presented here — see [FrKr.06]. 
entities are individuals, for any non-rigid structure can be extended to a rigid one. ${ }^{4}$

In other words, standard logic and mathematics describe individuals, yet we can simulate indiscernibility by using some tricks. Quantum mechanics does it by assuming symmetry postulates. Roughly speaking, only symmetric and anti-symmetric wavefunctions represent physical states, and the mean value of the measure of any observable does not change after a permutation of 'identical' quanta.

The Problem of Identity In Bohmian mechanics, identity is hidden. In such a mechanics particles are individuals in the above sense, having well defined positions and trajectories in any instant of time, and these confers them identity trough time. But position is a hidden variable, so it is identity (hence so it is the individuality of the particles). Here things happen more or less as the least elements of disjoined intervals of the reals mentioned above; we know that they are different, but we cannot express this difference in the theory. In Bohmian mechanics, the wavefunction $|\psi\rangle$ of a quantum system evolves according to the deterministic Schrödinger equation, but its actual positions obey a Guiding Equation, something that does not exist in the standard presentations of the theory. Bohmian mechanics shows that a quantum mechanics which accounts for all the phenomena of non-relativistic quantum mechanics and involving hidden variables is possible [Gol.09]. Thus, why not to keep with this solution?

Our reasons can be summed up as follows. David Hume regarded in his Treatise [Hum.85] the re-identification of objects in time (persons, say) as being consequence of habit (see [KrAr.06] for a resume of his ideas on this point). We could say, in parallel with his problem of induction (see the discussion on his Problem of Induction in [Pop.02]), that despite we attribute a continuity to the existence of a certain object, which by hypothesis is no more present to our senses, there seems to be no logical basis for we to assert that the object observed again and resembling that object which was present to our senses before, is the same object we have experienced before [Hum.85, Book I, Part IV, Section II]. In short, like induction, identity trough time has no rational justification, as we have seen above in the context of a quotation from Schrödinger in regarding quantum objects.

What about numerical identity? As usually conceived, numerical identity "is a relation everything has to itself and to nothing else" [Noo.09]. But, as Noonan observes, the notion of 'nothing else' presupposes numerical diversity, so this definition is redundant. Numerical identity is then defined either by Leibniz Law (in higher-order logics) or implicitly by reflexivity and substitutivity axioms in first-order logic (with the addition of the axiom of extensionality in set theories). But this is just TTI discussed above, and it entails that any object is an individual in the sense already discussed. If we regard this theory as valid for quantum objects, indiscernibility can be considered only as relative to some chosen attributes (relative indiscernibility), so either we take (as physicists do) this particular concept as standing for identity (contrariwise to TTI) or we need to accept the existence of hidden parameters which, yet not effectively, can confer numerical identity to the considered entities. Physics works quite well with each of these options, but the philosophy is bad with both of them, and no answer satisfies someone looking for a different metaphysics (see below). Identity is a useful concept in mathematics. It would be something completely strange if your number two was not the same as my number two when we are discussing

\footnotetext{
4. Rigid structures have only one automorphism, the identity function. Non-rigid structures may have other automorphisms, and two entities are indiscernible from the point of view of the structure if and only if there is an automorphism which leads one of them in the another. So, in a rigid structure, an object is indiscernible only from itself, according to TTI.
} 
arithmetics. No problem for in mathematics we (apparently) don't need to question TTI, yet we can do it for fun (and perhaps some application can be found later). But in the empirical sciences, if we assume TTI in the underlying logic, the only concept of indiscernibility we can use if that of relative indiscernibility, and so we will not be able to consider a metaphysical option which, following the forerunners of quantum theory, sees quantum objects as non-individuals, that is, as entities devoid of individuality (a bad name indeed, but with a long tradition) which, in our account, means to be devoid of identity. By the way, this is our way to approach the issue: we propose that the standard notion of identity (TTI) can be applied to objects in general, except in some particular cases. Our motivation, explained in full elsewhere, comes from Schrödinger, and we shall not touch this point again here (see [FrKr.06] for details).

Roughly speaking, non-individuals are entities that may have properties, may enter in relations with other non-individuals and with other objects. They can be aggregated in amounts, or collections having a cardinal, but when they are indiscernible (and sometimes they are not), no identification among them is possible even in principle, which means that no hidden parameters are presupposed to exist (and which confers them a radical difference to the least real numbers of our above example). Hence, non-individuals may or may not be indistinguishable; they are indistinguishable when partake all their properties and relations. But, since TTI does not hold, this does not entail that they are the very same object. In this case, they are absolutely indiscernible. Can we give some examples? Yes, we can. For instance, think of a BEC, a Bose-Einstein condensate. To get the general idea, let us quote an article from Scientific American (March 23, 2010): ${ }^{5}$

"In June 1995 our research group at the Joint Institute for Laboratory Astrophysics (now called JILA) in Boulder, Colo., succeeded in creating a minuscule but marvelous droplet. By cooling 2,000 rubidium atoms to a temperature less than 100 billionths of a degree above absolute zero (100 billionths of a degree kelvin), we caused the atoms to lose for a full 10 seconds their individual identities and behave as though they were a single 'superatom.' " [CoWi.10]

Of course we need to look this phrase with a grain of salt, but it exemplifies our previous example of the cricket balls. The claim that the rubidium atoms lose their individuality is completely in agreement with which has been accepted by theory in this case. As Wolfgang Ketterle, one of the Nobel Prize winners - shared with the authors of the above mentioned article - for the experimental discover of the BECs [Ket.07] says,

"[i]f we have a gas of ideal gas particles at high temperature, we may imagine those particles to be billiard balls (...). They race around in the container and occasionally collide. This is a classical picture. However, if we use the hypothesis of de Broglie that particles are matter waves, then we have to think of particles as wave packets. The size of a wave packet is approximately given by the de Broglie wavelength $\lambda_{d B}$, which is related to the thermal velocity $v$ of the particles as $\lambda_{d B}=h / m v$. Here $m$ is the mass of the particles and $h$ Plancks constant. Now, as long as the temperature is high, the wavepacket is very small and the concept of indistinguishability is irrelevant, because we can still follow the trajectory of each wavepacket and use classical concepts. However, a real crisis comes when the gas is cooled down: the colder the gas, the lower the velocity, and the longer the de Broglie wavelength. When individual wave packets overlap, then we have an identity crisis, because we can no

5. The interested reader will find interesting informations at an informal level at the BEC Homepage, http: //www.colorado.edu/physics/2000/bec/. 
longer follow trajectories and say which particle is which. At that point, quantum indistinguishability becomes important and we need quantum statistics." (op.cit.)

But, if we regard TTI as holding for the elements of a BEC, as we have seen above, we need either to accept the existence of hidden properties, that is, properties which would distinguish the elements from one each other, or their indiscernibility is only relative. The second alternative is not what physics tells us; the elements of a BEC are absolutely indiscernible. The first alternative is something we can do, but it is not in agreement with all interpretations which deny hidden variables. We prefer to investigate a third alternative, one which changes the metaphysics (and the ontology), a metaphysics of non-individuals, but which is compatible with the assumptions and with the formalism of quantum mechanics, both relativistic and non-relativistic (cf. [FrKr.06]).

Non-Reflexive QM As we have seen, the idea that quantum objects would be nonindividuals goes to some of the forerunners of quantum physics (see [FrKr.06] for a detailed discussion). When I speak of quantum objects (being them non-individuals of not), I am not necessarily guessing that quantum theory should be committed to particles in the usual sense of the world (small bodies having individuality). Really, quantum objects may be fields, propensities, whatever you wish. For instance, there is a long tradition (which goes at least to Schrödinger) in denying the existence of particles — see [Bit.07]. Paul Teller proposed to change the idea of $n$ particles by the notion of having a propensity do display $n$ quantum states (ibid.). But, we could ask: states of what? I tend to agree with Sunny Auyang in that "[p]hysical theories are about things" [Auy.95, p.152], and that even field theories may contain the means to refer. Thus, I am not discussing here the very nature of non-individuals, but just proposing that the very basic entities to which quantum theories refer, yet implicitly, could be treated as entities devoid of individuality. I have taken this idea quite seriously from the mathematical and logical points of view. Long time ago I began the development of a mathematics based on such an assumption, namely, seeing the basic objects in the sense that the notion of identity does not hold to them. In this theory, termed quasi-set theory (there are also some higher-logics based on the same motivations, termed 'Schrödinger logics' —cf. [FrKr.06]), there are collections (quasi-sets) having a cardinal, but not an associated ordinal. Thus we can regard more properly the collection of 2,000 rubidium atoms seen above as a quasi-set instead of a standard set of, say ZF (since, as it is well known, informally speaking, a set is a collection of distinguishable objects). In some recent works, my colleagues and I began the study of a quantum mechanics grounded on such formalism [DHKr.08], [DHKK.10], yet some ideas had been advanced before - see [FrKr.06] for the previous story.

In the remaining of this paper, I would like to advance some few ideas in a direction not touched yet, and which I intend to develop further in another work. In special, I propose to consider a quite radical idea already touched upon above, namely, that identity is just a useful concept, essential in mathematics, but which in the empirical sciences could be substituted (with certain advantages, as we have seen) by a weaker concept of indiscernibility. I found myself double motivated for proposing such an idea; firstly, I agree with Hume in that identity os objects can be ascribed only by habit, and I guess that this holds also for quantum objects (in agreement with some ideas advanced by Schrödinger when he still believed in particles; see [KrAr.06]). Secondly, my fears in stressing a so apparently crazy hypothesis were put aside when I found Wolfgang Pauli considering another quite strange idea (to our common sense view of the world grounded on classical physics), namely, that 
even macroscopic bodies could not have - at any time- a well-determined or even an almost determined position, and that the diffraction experiments on macroscopic objects would be conceivable in principle (cf. [d'Es.83, p.21]). Interesting enough, the diffraction phenomenum was recently realized with 'big' objects (big for the quantum scale), the $\mathrm{C}_{60}$ and $\mathrm{C}_{70}$ fullerene molecules [A.et.99]. Thus, standing on these giant's shoulders, I try to balance myself and look for an alternative approach to quantum theory, strongly grounded on non-individuality and on a non-classical logic. Since such a logic, namely, quasi-set theory, is a kind of non-reflexive logic, we call the corresponding quantum mechanics nonreflexive.

Here I can't develop the details, and this is of course not my intention either. But let me say only that within quasi-set theory we can develop all the mathematics we can build in ZF. So, all the standard formalism of quantum theory can be considered as done, and all we need to do is to define the relevant concepts which involve indiscernible non-individuals. We do it in terms of the Fock space formalism, by constructing two Fock spaces which differ by their inner products [DHKr.08]. The first is a Fock space for 'fermions', and the second for 'bosons'. Of course this terminology has no meaning from the mathematical point of view, but resemble the basic entities found in quantum theory, so we use them in the intended interpretation. In our Fock spaces, there is no labeled quanta in any step, so they are never 'identified' as individuals (say, by names or labels). The states refer only to certain operators which play the role of occupation number operators and permutations of quanta are unobservable (the mean value of the measure of any observable is the same before and after a permutation of two indiscernible non-individuals). We have also exemplified the use of this formalism to evaluate the correlations between the spin components of a twofermions system, explicitly showing that it is not necessary to first impose labels to the particles and then masking the individuation by a symmetrization postulate to obtain the usual results [DHKK.10].

Conclusion In this very short paper (maximum of 10 pages), I can't explore in details a so wide and and radical idea of questioning the standard notion of identity. But I hope the reader have understood the general motivation that, to me, induces the development of a non-reflexive quantum mechanics (NRQM). Let me end by emphasizing that this can of course be done within a framework such as $\mathrm{ZF}$, say by considering that the relevant relation is an equivalence relation (indiscernibility), and getting the formalism in a 'daydreamed' non-reflexive logic. But this version of the NRQM does not satisfy me for, as I have enlighten, it would be nothing more that standard quantum theory in sheep clothes, since identity continues to hold in the logical background and so only relative indistinguishability can be considered. What I propose is a radical change, by abandoning identity, and by basing NRQM on a different (non-classical) formal mathematical system, quasi-set theory. The full development of NRQM is still in its beginnings, but even if no new physical result will be achieved, at least from the conceptual point of view we have gains in understanding the role of identity and individuality in this realm, so as in general. In fact, identity is a so inlaid concept (interesting enough that logicians don't fear any more in questioning non-contradiction, the excluded middle and other ancient philosophical and metaphysical concepts ...) that apparently the only way of investigating its strength in quantum theory is by trying to find some other way of interpreting this theory in terms of some formalism which simply does not consider identity anyway. Let us see the future results. 


\section{References}

[Acz.01] Aczel, A. D. [2001], Entanglement: The Greatest Mystery in Physics, New York: Four Walls Eight Windows.

[A.et.99] Arndt, M., Nairz, O., Vos-Andreae, J., Keller, C., van der Zouw, G., ZeILINGER, A. [1999], 'Wave-particle duality of $\mathrm{C}_{60}$ molecules', Nature, V.401, 14 Oct. 1999, 680-2.

[Auy.95] Auyang, S. [1995], How is Quantum Field Theory Possible?, Princeton: Princeton Un. Press.

[Bit.07] BiтвоL, M. [2007], 'Schrödinger against particles and quantum jumps', in Evans, J. and Thorndike, A. S. (eds.), Quantum Mechanics at the Crossroads: New Perspectives from History, Philosophy and Physics, Berlin and Heidelberg: Springer, 81-106.

[CoWi.10] Cornell, E. A. and Wiemann, C. E. [2010], 'The Bose-EInstein Condensate', Scientific American, March 23.

[d'Es.83] D'Espagnat, B. [1983], In Search of Reality, New York: Springer.

[d'Es.03] D'Espagnat, B. [2003], Veiled Reality: An Analysis of Present-Day Quantum Mechanical Concepts, Westview Press.

[DHKr.08] Domenech, G., Holik, F. and Krause, D. [2008], Q-spaces and the foundations of quantum mechanics, Foundations of Physics 38 (11), 969-994.

[DHKK.10] Domenech, G., Holik, F., Kniznik, L, and Krause, D [2010]., 'No labeling quantum mechanics of indiscernible particles', Int. J. Theor. Phys. 49 (12), pp. 3085-3091.

[FrKr.06] French, S. \& Krause, D. [2006], Identity in Physics: A Historical, Philosophical, and Formal Analysis, Oxford: Oxford Un. Press.

[Gol.09] Goldstein, S. [2009], 'Bohmian Mechanics', The Stanford Encyclopedia of Philosophy (Spring 2009 Edition), http://plato.stanford.edu/archives/ spr2009/entries/qm-bohm.

[HuVi.10] Huggett, N. ANd Vistarini, T. [2010], 'Entanglement Exchange and Bohmian Mechanics', Manuscrito v. 33, n. 1, 223-242.

[Hum.85] Hume, D. [1985], Treatise of human nature, 2nd. Ed. L. A. Selby-Bigge. Oxford: Oxford Un. Press.

[Ket.07] KetTERLE, W. [2007], 'BoseĐEinstein condensation: identity crisis for indistinguishable particles', in Evans, J. and Thorndike, A. S. (eds.), Quantum Mechanics at the Crossroad: New Perspectives from History, Philosophy, and Physics, Berlin-Hiddelberg: Springer, pp.159-182.

[KrAr.06] Krause, D. and Arenhart, J.R.B. [2006], 'Hume, Schrödinger, e a individuação de objetos físicos', Revista Eletrônica Informação e Cognição, v.5, n.2, 59-71.

[Lad.07] Ladyman, J., Ross, D., Spurrett, D. and Collier, J. [2007], Every Thing Must Go: Metaphysics Naturalized, Oxford: Oxford Un. Press.

[Noo.09] Noonan, H. [2009], 'Identity', Stanford Encyclopedia of Philosophy, http: //plato.stanford.edu/entries/identity/.

[Pop.02] PopPer, K. R. [2002], The Logic of Scientific Discover, London and New Yourk: Routledge.

[Sch.09] Schiller, C. [2009], Motion Mountain: the Adventure of Physics, Part IV: Quantum Theory: The Smallest Change. Available at http://www. motionmountain.eu.

[Wei.93] Weinberg, S. [1993], Dreams of a Final Theory: The Search for the Fundamental Laws of Nature, London: Vintage. 